\title{
Difference in clinical presentation, immunology profile and treatment response of type 1 autoimmune hepatitis between United Kingdom and Singapore patients
}

\author{
Nwe Ni Than ${ }^{1,2}$ - Doreen Koay Siew Ching ${ }^{3} \cdot$ James Hodson $^{4} \cdot$ Patrick McDowell $^{1}$ • \\ Jake Mann $^{5} \cdot$ Ravi Gupta $^{2} \cdot$ Ennaliza Salazar ${ }^{3} \cdot$ Jing Hieng Ngu $^{3}$. \\ Ye Htun Oo ${ }^{1,2}$
}

Received: 27 October 2015/Accepted: 21 March 2016/Published online: 21 April 2016

(c) The Author(s) 2016. This article is published with open access at Springerlink.com

\begin{abstract}
Background Autoimmune hepatitis (AIH) is an immunemediated liver disease of unknown etiology. Increasing incidence of AIH in Asian patients has been reported. However, the phenotypic difference of Asian patients in Europe and Asia has still not been explored.

Aim To evaluate the clinical presentation, biochemical and immunological profiles, treatment response and survival outcome of type $1 \mathrm{AIH}$ from two tertiary liver transplant centres (United Kingdom and Singapore).

Method Patients who fulfilled the simplified diagnostic scoring criteria of AIH were included in the study. Patients with overlap syndrome were excluded.

Results Totals of 40 Asian patients and 159 Caucasian patients from the University Hospital of Birmingham National Health Service Foundation Trust, UK, were compared with 57 Asian patients from Singapore General Hospital, Singapore. Asian patients from Singapore present significantly much later (median 55 vs. 32 years, $p<0.001$ ), had higher MELD $(p<0.001)$ with lower albumin
\end{abstract}

Ye Htun Oo

y.h.oo@bham.ac.uk

1 Centre for Liver Research and NIHR Biomedical Research Unit in Liver Diseases, Institute of Immunology and Immunotherapy, University of Birmingham, Vincent Drive, Edgbaston, Birmingham B15 2TT, UK

2 Liver and Hepatobiliary Unit, Queen Elizabeth Hospital, Birmingham, UK

3 Department of Gastroenterology and Hepatology, Singapore General Hospital, Singapore, Singapore

4 Institute of Translational Medicine, Queen Elizabeth Hospital, Birmingham, UK

5 Leeds General Infirmary, Leeds, UK $(p<0.001)$ and higher bilirubin $(p<0.001)$ and lower ASMA positivity $(p<0.001)$ at diagnosis compared to UK Asian. Jaundice at presentation was much higher in Singapore Asian patients compared to UK Asian (53 vs. $30 \%$ ) but cirrhosis at diagnosis was more common in UK patients. Associated autoimmune conditions were less commonly seen in Singapore Asians. Comparing between UK cohorts, Asian patients present at younger age and have higher IgG level compared to Caucasian. Overall, 5-year transplant-free survival in all three cohorts was similar $(p=0.846)$.

Conclusion We demonstrate that AIH patients from Singapore present at older age with jaundice and have a low positivity of SMA. Despite these differences, transplant-free survival is similar in the two groups.

Keywords Autoimmune hepatitis - Asian - Caucasian · Ethnicity $\cdot$ Clinical features $\cdot$ Immunology $\cdot$ Survival

\section{Introduction}

Autoimmune hepatitis (AIH) is an immune-mediated liver disease of an unknown etiology with female preponderance [1]. It is a rare disease with a prevalence of $10-17$ per 100,000 populations in Europe and a mean incidence of 1-2 per 100,000 person-years [1,2]. Type $1 \mathrm{AIH}$ is characterised by the presence of anti-nuclear antibodies (ANA) and anti-smooth muscle antibodies (ASMA) in serum and it affects all ages, although the majority of cases are seen mainly in adults $[1,3]$. AIH is commonly associated with other autoimmune conditions.

It has been reported that ethnicity has an impact on the prevalence, clinical presentations, and the natural history of type 1 AIH [4-6]. Ethnicity-related prevalence of AIH was found to be significantly lower in Asians who reside in 
New Zealand when compared to Caucasians, despite being exposed to the same environment [7]. A previous study described that AIH in patients of African ethnicity presented with more aggressive disease at clinical presentation, were less likely to respond to conventional immunosuppressive treatment and resulted in worse liver morbidity-related outcomes compared to Caucasians [8]. Asian-American AIH patients also demonstrated more aggressive disease with poorer survival, and Hispanic populations had a higher prevalence of biopsy-proven cirrhosis at presentation compared to Caucasians [9]. A study in the UK in 2002 showed that non-European Caucasians (African, Asian and Arabic) presented with more severe forms of liver disease in all aspects with international criteria for a diagnosis of $\mathrm{AIH}$, and they seemed to require higher levels of immunosuppression from earlier points after diagnosis [10]. Another study by Verma et al. [8] mentioned that black ethnicity, especially men, have more aggressive disease, and that they are less likely to respond to standard immunosuppression with a worse outcome than non-black ethnicity. Taken together, these reports suggest that ethnicity has an impact on the natural history of AIH.

In this study, we aim to investigate the difference in natural history, mode of presentation, immunological profiles, associated autoimmune diseases, response to immunosuppression and survival outcomes of Asian type 1 AIH who reside in two different continents; namely the United Kingdom (UK) and Singapore. In addition, we compare a Caucasian patient cohort to an Asian cohort within the same hospital in the UK.

\section{Patients and methods}

\section{Study cohort}

The details of patients with type 1 AIH who attended liver outpatients at two tertiary liver transplant centres: University Hospital of Birmingham, UK, and Singapore General Hospital, Singapore, were retrospectively collected and analysed. Data was obtained between the years 1995 and 2015 in the UK and between 2001 and 2014 in Singapore.

The UK cohorts were drawn from 203 patients with type 1 AIH. Of these, 40 patients of Asian ethnicity were included in the study and compared with 57 patients from Singapore. An additional cohort of 159 Caucasian patients from the same UK hospital was also compared to the UK Asian cohort. The remaining four patients from the UK were of different ethnicities and were not considered in this analysis. The total duration of follow-up was 10 years (median of 4 years, range 1 month to 18 years) for the UK patient cohort and 14 years for the Singapore patient cohort (median of 4 years, range 1 month to 11 years).
All patients were evaluated and diagnosed with type $1 \mathrm{AIH}$ by hepatologists within our medical centres based on clinical, biochemical, and immunological parameters along with liver histology. All liver biopsies were reviewed and reported by dedicated liver histopathologists. Patients with a simplified $\mathrm{AIH}$ score of greater than or equal to six were included in the study. Patients with overlap syndrome (primary biliary cirrhosis or primary sclerosing cholangitis with type $1 \mathrm{AIH}$ ) were excluded from the study. Other liver conditions, such as metabolic liver diseases, viral hepatitis, alcoholic or non-alcoholic fatty liver diseases, were also excluded.

Data were collected thoroughly from electronic case notes, clinical letters and treatment charts. Demographics data, clinical presentations, blood parameters such as biochemistry and immunology, liver histology and the presence of other associated autoimmune conditions were documented. Treatment of autoimmune hepatitis such as usage of the steroid, azathioprine, other second-line immunosuppression and liver-related complications such as decompensation and development of hepatocellular carcinoma were also documented.

\section{Statistical analysis}

Comparisons were made between the UK Asian cohort and both the Singapore Asian and UK Caucasian cohorts. Since the UK Asian cohort was included in both these analyses, all $p$ values were Bonferroni-adjusted for two comparisons, to help control the type 1 error rate.

Dichotomous variables were compared between the cohorts using Fisher's exact tests, with ordinal and continuous variables assessed using Mann-Whitney tests, and reported as medians and interquartile ranges (IQRs). The number of flareups were converted into rates per patient-year, in order to account for the differences in follow-up durations between the patients, with comparisons between the cohorts performed using the OpenEpi calculator [11]. Transplant-free survival was assessed using Kaplan-Meier curves, with a log-rank test used to compare between the cohorts.

All analyses were performed using IBM SPSS Statistics 22 (IBM, Armonk, NY, USA), with the exception of the comparison of flare-up rates. Patients with missing data were excluded on a per-analysis basis and $p<0.05$ was deemed to be indicative of statistical significance throughout.

\section{Results}

\section{Comparison between UK Caucasian and UK Asian AIH patient cohorts}

A total of 159 Caucasian patients were compared against 40 Asian patients from the same hospital in the UK. The 
details of the comparison are shown in Tables 1 and 2 . The majority of the patients were female (79\% in Caucasian vs. $75 \%$ in Asian, $p=1.000$ ), with Caucasian patients presenting with the disease at significantly later ages than Asians (median age of 51 vs. 32 years, $p<0.001$ ). BMI was similar in the two groups, with a median of 26.2 in Caucasian versus 26.0 in Asian $(p=0.556)$. No significant differences were detected between the groups in the rates of hypertension $(p=0.648)$, type 2 diabetes $(p=0.442)$ or hypercholesterolemia $(p=0.776)$.

The most commonly observed type of associated autoimmune conditions were connective tissue disorders, (vasculitis, systemic lupus erythromatus, limited scleroderma, Sjogren's disease, vitiligo, psoriasis or rheumatoid disorder), which affected similar proportions of Asians and Caucasians ( 18 vs. $21 \%, p=1.000$ ). The only type of associated autoimmune conditions that differed significantly by ethnicity was those that were haematology- related (e.g. autoimmune hemolytic thrombocytopenia or autoimmune hemolytic anemia), which were present in $15 \%$ of Asians, compared to $3 \%$ of Caucasian patients $(p=0.020)$.

Rates of liver biopsy at the time of diagnosis were similar in the Asian and Caucasian groups (73 vs. $75 \%$, $p=1.000$ ), as were the rates of cirrhosis (40 vs. $51 \%$, $p=0.576)$. Hepatocellular carcinoma was uncommon in both cohorts, with no cases in Asian patients and only 3 $(2 \%)$ in Caucasians. Around $10 \%$ of patients in both cohorts presented with features of liver decompensation such as ascites, hepatic encephalopathy or variceal bleed at the time of diagnosis. The proportions of patients presenting with jaundice were similar in both groups (21 in Caucasian vs. $30 \%$ in Asian, $p=0.424$ ). The models for end-stage liver disease (MELD) score were also similar in the two cohorts (median 6 in Caucasian vs. 7 in Asian, $p=0.324)$. At diagnosis, both Caucasian and Asian

Table 1 Demographic and clinical comparison between the three cohorts

\begin{tabular}{|c|c|c|c|c|c|}
\hline Factor & $\begin{array}{l}\text { UK Asian } \\
(n=40)\end{array}$ & $\begin{array}{l}\text { Singapore Asian } \\
(n=57)\end{array}$ & $p$ value* & $\begin{array}{l}\text { UK Caucasian } \\
(n=159)\end{array}$ & $p$ value** \\
\hline \multicolumn{6}{|l|}{ Demographics } \\
\hline Age at start of follow-up & $32.0(22.0-52.4)$ & $55.0(50.0-64.0)$ & $<0.001$ & $50.5(28.9-60.3)$ & 0.002 \\
\hline Gender (female) & $30(75 \%)$ & $49(86 \%)$ & 0.386 & $125(79 \%)$ & 1.000 \\
\hline Body mass index & $26.0(22.3-28.0)$ & $24.7(21.2-28.1)$ & 0.580 & $26.2(23.4-31.5)$ & 0.556 \\
\hline Biopsied at diagnosis & $29(73 \%)$ & $56(98 \%)$ & $<0.001$ & $119(75 \%)$ & 1.000 \\
\hline Cirrhosis at diagnosis & $16(40 \%)$ & $12(23 \%)$ & 0.154 & $81(51 \%)$ & 0.576 \\
\hline Hepatocellular carcinoma & $0(0 \%)$ & $1(2 \%)$ & 1.000 & $3(2 \%)$ & 1.000 \\
\hline Hypertension & $8(20 \%)$ & $24(42 \%)$ & 0.058 & $45(28 \%)$ & 0.648 \\
\hline Type two diabetes & $9(23 \%)$ & $10(18 \%)$ & 1.000 & $22(14 \%)$ & 0.442 \\
\hline Hypercholesterolemia & $3(8 \%)$ & $16(28 \%)$ & 0.036 & $6(4 \%)$ & 0.776 \\
\hline \multicolumn{6}{|l|}{ Clinical presentation } \\
\hline Jaundice & $12(30 \%)$ & $30(53 \%)$ & 0.074 & $33(21 \%)$ & 0.424 \\
\hline Decompensated at diagnosis & $4(10 \%)$ & $2(4 \%)$ & 0.452 & $15(9 \%)$ & 1.000 \\
\hline \multicolumn{6}{|l|}{ Bloods at diagnosis } \\
\hline Albumin & $40(31-43)$ & $31(27-35)$ & $<0.001$ & $39(34-43)$ & 1.000 \\
\hline Bilirubin & $19(11-38)$ & $49(18-172)$ & $<0.001$ & $20^{\mathrm{a}}(11-38)$ & 1.000 \\
\hline INR & $1.1(1.0-1.3)$ & $1.2(1.1-1.3)$ & 0.606 & $1.1(1.0-1.2)$ & 0.636 \\
\hline Model for end stage liver disease (MELD) & $7(6-10)$ & $13(9-18)$ & $<0.001$ & $6(6-10)$ & 0.324 \\
\hline \multicolumn{6}{|l|}{ Other autoimmune conditions } \\
\hline Any gastro-intestinal & $5(13 \%)$ & $0(0 \%)$ & 0.020 & $12(8 \%)$ & 0.688 \\
\hline Any connective & $7(18 \%)$ & $6(11 \%)$ & 0.746 & $33(21 \%)$ & 1.000 \\
\hline Any endocrine & $3(8 \%)$ & $1(2 \%)$ & 0.606 & $19(12 \%)$ & 1.000 \\
\hline Any renal & $2(5 \%)$ & $0(0 \%)$ & 0.336 & $1(1 \%)$ & 0.208 \\
\hline Any haematology & $6(15 \%)$ & $1(2 \%)$ & 0.036 & $5(3 \%)$ & 0.020 \\
\hline
\end{tabular}

Continuous variables are reported as medians and interquartile ranges, with numbers and rates quoted otherwise

$p$ values are post hoc comparisons between the UK Asian* Singapore Asian or ** UK Caucasian cohorts. Mann-Whitney tests and Fisher's exact tests were used, as applicable, with Bonferroni adjustment for two comparisons applied to the resulting $p$ values. $p$ values significant at $p<0.05$ are shown in bold

${ }^{\text {a }}$ Based on $n=32$, due to missing data 
Table 2 Comparison between UK Caucasian and UK Asian: Immunology and treatment

\begin{tabular}{|c|c|c|c|c|c|}
\hline Factor & $\begin{array}{l}\mathrm{UK} \\
\text { Asian } \\
(n=40)\end{array}$ & $\begin{array}{l}\text { Singapore Asian } \\
(n=57)\end{array}$ & $p$ value* & $\begin{array}{l}\text { UK Caucasian } \\
(n=159)\end{array}$ & $p$ value** \\
\hline \multicolumn{6}{|l|}{ Immunology profile } \\
\hline Anti-nuclear antibody & $32(80 \%)$ & $53(93 \%)$ & 0.134 & $121(76 \%)$ & 1.000 \\
\hline Anti-neutrophil cytoplasmic antibody & $5(13 \%)$ & $2(4 \%)$ & 0.248 & $30(19 \%)$ & 1.000 \\
\hline Anti-smooth muscle (type 1) antibody & $29(73 \%)$ & $16(28 \%)$ & $<0.001$ & $125(79 \%)$ & 0.810 \\
\hline Soluble liver antigen & $0(0 \%)$ & $1(2 \%)$ & 1.000 & $0(0 \%)$ & 1.000 \\
\hline Ig G & $21.9(15.5-30.6)$ & $25.0(16.7-33.3)$ & 0.832 & $17.8(12.7-24.7)$ & $\mathbf{0 . 0 3 2}$ \\
\hline $\operatorname{Ig} \mathrm{M}$ & $1.9(1.2-2.5)$ & $1.5(1.1-3.1)$ & 1.000 & $1.6(1.1-2.5)$ & 0.700 \\
\hline Ig A & $4.0(2.1-5.2)$ & $4.1(3.2-5.6)$ & 0.342 & $2.9(1.9-4.3)$ & 0.079 \\
\hline \multicolumn{6}{|l|}{ Medications at diagnosis } \\
\hline Steroids & $37(93 \%)$ & $55(98 \%)$ & 0.610 & $135(85 \%)$ & 0.604 \\
\hline Azathioprine & $20(50 \%)$ & $19(33 \%)$ & 0.282 & $79(50 \%)$ & 1.000 \\
\hline Mycophenolate mofetil & $6(15 \%)$ & $0(0 \%)$ & 0.008 & $12(8 \%)$ & 0.424 \\
\hline \multicolumn{6}{|l|}{ Current medications } \\
\hline Steroids & $30(75 \%)$ & $36(63 \%)$ & 0.542 & $109(69 \%)$ & 1.000 \\
\hline Azathioprine & $25(63 \%)$ & $19(33 \%)$ & 0.014 & $91(57 \%)$ & 1.000 \\
\hline Mycophenolate mofetil & $7(18 \%)$ & $0(0 \%)$ & 0.003 & $28(18 \%)$ & 1.000 \\
\hline Number of flare-ups (per patient-year) ${ }^{a}$ & $0.33^{\mathrm{a}}(0.26-0.41)$ & $0.22^{\mathrm{a}}(0.17-0.29)$ & $0.062^{\mathrm{a}}$ & $0.24^{\mathrm{a}}(0.22-0.28)$ & $0.060^{\mathrm{a}}$ \\
\hline Concerns with compliance & $6(15 \%)$ & $0(0 \%)$ & 0.008 & $15(9 \%)$ & 0.770 \\
\hline
\end{tabular}

Continuous variables are reported as medians and interquartile ranges, with numbers and rates quoted otherwise

$p$ values are post hoc comparisons between the UK Asian and * Singapore Asian or ** UK Caucasian cohorts. Mann-Whitney tests and Fisher's exact tests were used, as applicable, with Bonferroni adjustment for two comparisons applied to the resulting $p$ values. $p$ values significant at $p<0.05$ are shown in bold

${ }^{a}$ Reported as rates per patient-year and $95 \%$ confidence intervals, with $p$ values from the mid- $P$ exact test

patients had comparable levels of albumin (median 39 vs. $40, p=1.000)$, bilirubin (20 vs. $19, p=1.000)$ and INR (1.1 vs. $1.1, p=0.636)$.

The majority of the patients had anti-nuclear antibody positivity at diagnosis, followed by positive anti-smooth muscle antibody (ASMA) and anti-neutrophil cytoplasmic antibodies (ANCA), with all three having similar rates in both groups. Immunoglobulin-G (Ig G) was found to be significantly higher in Asian cohort (median 21.9 vs. 17.8, $p=0.032$ ).

The majority of the patients in both groups were started on steroids followed by azathioprine (AZA) and mycophenolate mofetil (MMF), with the treatment rates in both groups being similar (all, $p=1.000$ ). There were only around a $20 \%$ point reduction in use of steroids in both cohorts from the time of diagnosis to the most recent follow-up. The proportion of patients for whom there were concerns about compliance was $9 \%$ in Caucasians and $15 \%$ in Asians $(p=0.770)$. The documented number of flare-ups per year were low in both groups during followup (0.24 vs. 0.33 episodes per patient year). Transplant-free survival was also similar in the two groups, with rates at 5 years of $88 \%$ in Caucasians compared to $84 \%$ in Asians, $(p=1.000$, Fig. 1).

\section{Comparison between UK Asian and Singapore Asian cohort of AIH patients}

The 40 UK Asian patients were then compared to a cohort of 57 Asian patients from Singapore. The Singapore cohort presented at a significantly older age than the UK Asians (median 55 vs. 32 years, $p<0.001$ ), and were significantly more likely to be biopsied at diagnosis (98 vs. $73 \%$, $p<0.001)$. No significant differences were detected in the gender $(p=0.386)$ or BMI $(p=0.580)$ distributions of the cohorts nor in the rates of type 2 diabetes $(p=1.000)$. However, Singapore Asians had significantly higher rates of hypercholesterolemia ( 28 vs. $8 \%, p=0.036$ ) than UK Asians. Jaundice (53 vs. $30 \%, p=0.074$ ) and hypertension (42 vs. $20 \%, p=0.058$ ) were also more common in Singapore Asians, although not significantly so.

Of the blood work taken at diagnosis, the patients from Singapore had significantly lower albumin (median 31 vs. $40, p<0.001$ ), and significantly higher bilirubin (49 vs. 19, $p<0.001)$ and MELD (13 vs. 7, $p<0.001)$ than Asians from the UK. Associated autoimmune conditions were less commonly seen in Singapore Asians, with significantly lower rates of gastro-intestinal- (0 vs. $13 \%$, $p=0.020)$ and hematology-related (2 vs. $15 \%$, 


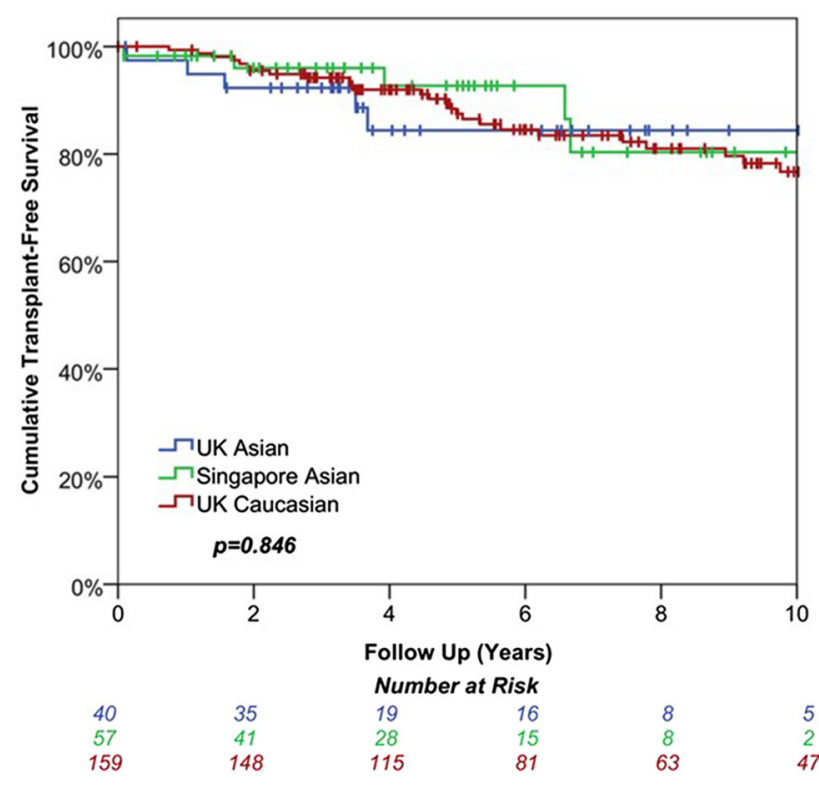

Fig. 1 Kaplan-Meier survival curves of all study cohorts

$p=0.036)$ conditions than Asians from the UK. Analysis of the immunological profiles of the two groups generally found no significant differences, with the exception of antismooth muscle antibody, which was detected in $28 \%$ of Singapore Asians, compared to $73 \%$ of those from the UK $(p<0.001)$.

Steroid usage was similar in the two groups at baseline (Singapore: 98 vs. UK: $93 \%, p=0.610$ ) and the most recent follow-up (75 vs. $63 \%, p=0.542$ ). Azathioprine was less commonly used in Singapore, with usage rates of 33 versus $50 \%(p=0.282)$ at diagnosis, and 33 versus $63 \%(p=0.014)$ at the most recent follow-up. None of the Singapore Asians used MMF, compared to $15 \%$ of UK Asians at diagnosis and $18 \%$ at the most recent follow-up ( $p=0.008,0.003)$. There were no concerns with compliance in the Singapore cohort, compared to $15 \%$ of the UK Asian cohort $(p=0.008)$.

Transplant-free survival was similar in the two groups $(p=1.000)$, with rates at 5 years of $93 \%$ in Singapore and $84 \%$ in UK Asians ( $p=1.000$, Fig. 1). Flare-up rates were low in both groups, with an average of 0.22 per patient-year in Singapore Asians, compared to 0.33 in those from the UK $(p=0.062)$.

\section{Discussion}

Ethnic difference has been reported to have an impact on the natural history of AIH $[4-6,8,12]$; however, the difference in clinical phenotype of Asian patients between different continents is still unexplored. In this study, we demonstrated differences in age at initial diagnosis, clinical presentation, immunology profiles, associated autoimmune conditions, immunosuppression used and treatment response/compliance, between Asian type 1 AIH patients in UK and Singapore.

Autoimmune hepatitis could present at any age but generally peaks around puberty and between the 4th and 6th decades in adult life $[6,13]$. In our study, UK Caucasian patients presented at a significantly later age compared to the UK Asian population. From the comparisons with the Singapore Asian cohort, we observed that the UK Asian patients' cohort presented earlier, on an average during their 3 rd decade, compared to the 5 th decade of the cohort from Singapore. This later presentation may explain the reason of higher incidence of hypertension and hypercholesterolemia in the Singapore Asian AIH cohort. AIH patients are generally asymptomatic, and diagnosis was made from routine blood tests, although jaundice at initial presentation is not uncommon. Interestingly, we noted that Singapore Asian patients commonly presented with jaundice, and had significant higher levels of bilirubin, a higher MELD score, and lower levels of albumin at diagnosis.

Immunological parameters such as hyperglobulinemia and positive autoantibodies are crucial for the diagnosis of type 1 AIH [14]. Type 1 AIH is characterised by the presence of ANA and/or SMA, although $19 \%$ of AIH patients may not have any evidence of serological positivity at the time of presentation [15]. Low levels of SMA positivity have been previously described in Asian patients with type 1 AIH [16] and we have seen that in our Singapore Asian cohort. A large proportion of UK-Asian patients in our study were cirrhotic (40\%), thus there was a potential early onset of disease in the UK cohort, which may be attributable to a higher index of diagnosis related to higher SMA positivity on immunological investigation. UK Caucasian patients expressed lower levels of Ig-G compared to the Asian cohort, and were less likely to have associated hematological autoimmune conditions. None of the other factors compared between UK Asian and UK Caucasian were found to differ significantly, including transplant-free survival.

In addition, associated autoimmune diseases such as coeliac disease and inflammatory bowel disease are more common in UK Asian patients than those from Singapore. Previous observations have suggested that Th17 cells and memory mucosa lymphocytes are involved in gut-liver axis immunology [17, 18], and the high prevalence of inflammatory bowel disease in western countries may be the reason for the association.

Despite the availability of effective treatment, AIH is not a benign condition, with recent long-term studies reporting a twofold higher mortality than that of the general population $[19,20]$. The majority of patients with AIH usually respond to standard immunosuppressive therapy 
with steroids and azathioprine [AZA] [21], which we observed in our cohort. Around $80 \%$ of patients achieved remission with standard immunosuppressive therapy [22, 23], while $10-15 \%$ of patients do not achieve biochemical remission with these standard therapies [24-26]. Alternative immunosuppression such as MMF could be used for those who cannot tolerate AZA [27]. Our study found that MMF was used as second-line therapy only in UK patients. All patients from Singapore tend to be controlled by standard immunosuppression with steroids and azathioprine.

End-stage liver cirrhosis related to AIH accounts for 4-6\% of adult liver transplantation in Europe and the United States $[28,29]$. Of patients with AIH, $23 \%$ from Singapore and $40 \%$ of Asians and $51 \%$ of Caucasians in the UK were cirrhotic at the time of presentation. Wong and colleagues reported that $\mathrm{AIH}$ in Asian patients who reside in United States tend to present with more aggressive states and patients are cirrhotic at the time of presentation [30]. In our study, the index incidence of cirrhosis at diagnosis was higher among UK Asian patients, which may be related to earlier onset or a high index of suspicion or early referral of this group to tertiary transplant centres.

Wong et al. [30] also suggested that INR tends to be higher in Asian population. However, we did not notice any difference in albumin titre and INR between the UK Asians and Caucasians in our study, as the majority of cases are compensated Child-Pugh grade A cirrhosis. Only $10 \%$ of UK Asian patients and $4 \%$ of Singapore patients presented with decompensated liver disease. It is possible that differences in health care provision between different studies may have impacted on the synthetic function and timing of presentation. Long-term liver-related morbidity such as decompensation or hepatocellular carcinoma incidence of both groups remained similar. Higher MELD score was observed in Singapore Asian patients, which could be related to higher bilirubin in this cohort, as the initial presentation in more than half of this cohort was with jaundice. Our study importantly demonstrated similar transplant-free survival in both groups.

We have identified a few limitations associated with our study, mainly due to the nature of retrospective and descriptive studies. First, we compared our UK Asians with Singapore Asians. The majority of UK Asian cohorts were of Indian origin while Singapore Asians were of Far East origin. Secondly, our cohort might represent AIH patients with more severe disease since we were tertiary liver transplant centres. Diet might also play an important role in differences between our cohorts. There is a populationbased study conducted in New Zealand [31] that showed alcohol consumption was associated with a lower risk of diagnosing with $\mathrm{AIH}$, but individuals who were vegetarian for more than a year and had antibiotics usage within 12 months before AIH diagnosis were significant risk factors associated with AIH.
In conclusion, our data suggested that AIH patients from Singapore present at older age with jaundice and have a low positivity of SMA with similar survival outcomes. These findings highlight that future work is required to explore the ethnicity-related epigenetic, microbiome, environmental and dietary factors for further understanding of AIH immunopathogenesis. We aim to conduct a large number multiple-centre study in the near future to have a better understanding of the impact of ethnicity in autoimmune hepatitis.

Acknowledgements We acknowledge the funding bodies MRC and NIHR.

\section{Compliance with ethical standards}

Funding This study is supported by the National Institute for Health Research (NIHR) Liver Biomedical Research Unit based at the University Hospital Birmingham NHS Trust and the University of Birmingham, UK. The views expressed are those of the author(s) and not necessarily those of the NHS, the NIHR or the Department of Health. The data from Singapore were supported by gastroenterologists and hepatologists from Singapore.

\section{Conflict of interest None.}

Ethical approval All procedures performed in studies involving human participants were in accordance with the ethical standards of the institutional and/or national research committee and with the 1964 Helsinki declaration and its later amendments or comparable ethical standards.

Informed consent Informed consent was obtained from all individual participants included in the study.

Grants and financial support Ye Htun Oo was supported by Medical Research Council clinician scientist fellowship funding, NIHR Liver BRU and UHB Charity. The National Institute of Health Research (NIHR) Liver Biomedical Research Unit (BRU) at University Hospital Birmingham NHS Trust and University of Birmingham funded Nwe Ni Than.

Open Access This article is distributed under the terms of the Creative Commons Attribution 4.0 International License (http://crea tivecommons.org/licenses/by/4.0/), which permits unrestricted use, distribution, and reproduction in any medium, provided you give appropriate credit to the original author(s) and the source, provide a link to the Creative Commons license, and indicate if changes were made.

\section{References}

1. Zachou K, Muratori P, Koukoulis GK, Granito A, Gatselis N, Fabbri A, et al. Review article: autoimmune hepatitis-current management and challenges. Aliment Pharmacol Ther 2013;38(8):887-913

2. Makol A, Watt KD, Chowdhary VR. Autoimmune hepatitis: a review of current diagnosis and treatment. Hepat Res Treat 2011;2011:390916

3. Oo YH, Hubscher SG, Adams DH. Autoimmune hepatitis: new paradigms in the pathogenesis, diagnosis, and management. Hep Intl 2010;4(2):475-493 
4. Czaja EOS AJ, Bittencourt PL, Cancado ELR, Porta G, Goldberg AC, Donaldson PT. Clinical distinctions and pathogenic implications of type 1 autoimmune hepatitis in Brazil and the United States. J Hepatol 2002;37:302-308

5. Hassan N, Siddiqui AR, Abbas Z, Hassan SM, Soomro GB, Mubarak M, et al. Clinical profile and HLA typing of autoimmune hepatitis from Pakistan. Hepat Mon 2013;13(12):e13598

6. Lim KN, Casanova RL, Boyer TD, Bruno CJ. Autoimmune hepatitis in African Americans: presenting features and response to therapy. Am J Gastroenterol 2001;96(12):3390-3394

7. Ngu JH, Bechly K, Chapman BA, Burt MJ, Barclay ML, Gearry RB, et al. Population-based epidemiology study of autoimmune hepatitis: a disease of older women? J Gastroenterol Hepatol 2010;25(10):1681-1686

8. Verma S, Torbenson M, Thuluvath PJ. The impact of ethnicity on the natural history of autoimmune hepatitis. Hepatology 2007;46(6):1828-1835

9. Wong RJ, Gish R, Frederick T, Bzowej N, Frenette C. The impact of race/ethnicity on the clinical epidemiology of autoimmune hepatitis. J Clin Gastroenterol 2012;46(2):155-161

10. Zolfino T, Heneghan MA, Norris S, Harrison PM, Portmann BC, McFarlane IG. Characteristics of autoimmune hepatitis in patients who are not of European Caucasoid ethnic origin. Gut 2002;50(5):713-717

11. Dean AG, Soe MM. OpenEpi: open source epidemiologic statistics for public health, version 3.03a (updated 2015/05/04). www.OpenEpi.com.

12. Gronbaek L, Vilstrup H, Jepsen P. Autoimmune hepatitis in Denmark: incidence, prevalence, prognosis, and causes of death. A nationwide registry-based cohort study. J Hepatol 2014;60(3): 612-617

13. Heneghan MA, Yeoman AD, Verma S, Smith AD, Longhi MS. Autoimmune hepatitis. Lancet 2013;382(9902):1433-1444

14. Alvarez F, Berg PA, Bianchi FB, Bianchi L, Burroughs AK, Cancado EL, et al. International autoimmune hepatitis group report: review of criteria for diagnosis of autoimmune hepatitis. J Hepatol 1999;31(5):929-938

15. Mehendiratta V, Mitroo P, Bombonati A, Navarro VJ, Rossi S, Rubin R, et al. Serologic markers do not predict histologic severity or response to treatment in patients with autoimmune hepatitis. Clin Gastroenterol Hepatol 2009;7(1):98-103

16. Zhao P, Han Y. Low incidence of positive smooth muscle antibody and high incidence of isolated IgM elevation in Chinese patients with autoimmune hepatitis and primary biliary cirrhosis overlap syndrome: a retrospective study. BMC Gastroenterol 2012;12:1

17. Oo YH, Banz V, Kavanagh D, Liaskou E, Withers DR, Humphreys E, et al. CXCR3-dependent recruitment and CCR6- mediated positioning of Th-17 cells in the inflamed liver. J Hepatol 2012;57(5):1044-1051

18. Eksteen B, Mora JR, Haughton EL, Henderson NC, Lee-Turner L, Villablanca EJ, et al. Gut homing receptors on CD8 T cells are retinoic acid dependent and not maintained by liver dendritic or stellate cells. Gastroenterology 2009;137(1):320-9. doi:10.1053/ j.gastro.2009.02.046

19. Ngu JH, Gearry RB, Frampton CM, Stedman CA. Mortality and the risk of malignancy in autoimmune liver diseases: a population-based study in Canterbury, New Zealand. Hepatology 2012;55(2):522-9. doi:10.1002/hep.24743

20. Hoeroldt B, McFarlane E, Dube A, Basumani P, Karajeh M, Campbell MJ, et al. Long-term outcomes of patients with autoimmune hepatitis managed at a nontransplant center. Gastroenterology 2011;140(7):1980-1989

21. Eksteen B, Liaskou E, Adams DH. Lymphocyte homing and its role in the pathogenesis of IBD. InflammBowelDis 2008

22. Czaja AJ. Advances in the current treatment of autoimmune hepatitis. Dig Dis Sci 2012;57(8):1996-2010

23. Liberal R, Grant CR, Mieli-Vergani G, Vergani D. Autoimmune hepatitis: a comprehensive review. J Autoimmun 2013;41: 126-139

24. Vierling JM, Flores PA. Evolving new therapies of autoimmune hepatitis. Clin Liver Dis 2002;6(3):825-850

25. Schramm C, Weiler-Normann C, Wiegard C, Hellweg S, Muller S, Lohse AW. Treatment response in patients with autoimmune hepatitis. Hepatology 2010;52(6):2247-2248

26. Czaja AJ, Freese DK. Diagnosis and treatment of autoimmune hepatitis. Hepatology 2002;36(2):479-497

27. Hennes EM, Oo YH, Schramm C, Denzer U, Buggisch P, Wiegard $\mathrm{C}$, et al. Mycophenolate Mofetil as second line therapy in autoimmune hepatitis? Am J Gastroenterol 2008

28. Seaberg EC, Belle SH, Beringer KC, Schivins JL, Detre KM. Liver transplantation in the United States from 1987-1998: updated results from the Pitt-UNOS Liver Transplant Registry. Clin Transpl 1998;17-37

29. Adam R, McMaster P, O'Grady JG, Castaing D, Klempnauer JL, Jamieson N, et al. Evolution of liver transplantation in Europe: report of the European Liver Transplant Registry. Liv Transpl 2003;9(12):1231-1243

30. Wong RJ, Giss R, Frederick T, Bzowej N, Frenette C, Zachou K. The impact of race/ethnicity on the clinical epidemiology of autoimmune hepatitis. J Clin Gastroenterol 2012;46:155-161

31. Ngu JH, Gearry RB, Frampton CM, Stedman CA. Autoimmune hepatitis: the role of environmental risk factors: a populationbased study. Hepatol Int 2013;7(3):869-875 